\title{
Intelligent Routing and Flow Control in MANETs
}

\author{
M. Belkadi ${ }^{1}$, M. Lalam ${ }^{1}$, A. M'zoughi ${ }^{2}$, N. Tamani ${ }^{1}$, M. Daoui $^{1}$ \\ and R. Aoudjit ${ }^{1}$ \\ ${ }^{1}$ Computer Science Department, Tizi Ouzou University, Algeria \\ ${ }^{2}$ IRIT UPS 118, Toulouse Cedex, France
}

\begin{abstract}
Improving the performance of Mobile Ad hoc NET-works (MANETs) is challenging. The MANETs communications are presented with many constraints such as dynamic topology, unreliability of wireless links, and limited bandwidth and energy resources. The routing in MANETs can be particularly difficult due to aggravated congestion problem. This paper proposes a new QoS (Quality of Service) routing protocol combined with the flow control mechanism. This routing protocol selects the routes having more resources in an intelligent manner and does not rely on diffusion. The proposed routing protocol uses a new metric to find the route with higher transmission rate, less latency and better stability. In order to reinforce the congestion avoidance, a flow control mechanism is employed to adapt the transmission rate for each route. The proposed routing solution is modeled by ant systems. The numerical results are obtained using the Network Simulator (NS2.31). These results confirm that the joint consideration of flow control and routing can significantly improve the network performance compared to other QoS routing protocols such as the QoS-AODV (Ad hoc On Demand Vector with QoS) protocol.
\end{abstract}

Keywords: MANETs, QoS routing, flow control, congestion control, ant systems

\section{Introduction}

A mobile ad hoc network (MANET) is an autonomous system of wireless mobile nodes without any fixed infrastructures. This kind of network promises many advantages in terms of cost and flexibility compared to network with infrastructures. MANETs are very suitable for a great variety of applications such as data collection, seismic activities, medical applications. . . Unfortunately nodes in MANETs are limited in energy, bandwidth... These resources constraints pose a set of non trivial problems; in particular, routing and flow control [1].

Routing in communication networks is necessary because, generally, nodes are not directly connected. The main problem solved by any routing protocol is to direct traffic from sources to destinations, but nowadays, because of increasing complexity in modern networks, routing algorithms face important challenges.

The routing function is particularly challenging in these networks because the network structure is constantly changing and the network resources are limited. This is particularly true in wireless ad hoc networks where node mobility and link failures produce constant changes in the network topology. Routing algorithms lack of adaptability to frequent topological changes, limited resources, energy availability. . . reduces network performance.

Traditional routing algorithms proposed for MANETs in the literature, such as AODV [2], DSR [3] and OLSR [4] route data without considering explicitly Quality of Service (QoS) constraints. The number of hops is the most common criterion adopted by such proposed routing protocols. These algorithms are inappropriate to applications requiring a quality of service. The new requirements in QoS for mobile applications impose routes that satisfy end to end QoS with sufficient available resources [5].

On the other hand, flow control is another major challenge in networks research that adapts transmission rate to available resources capacities in order to avoid congestion. It is necessary 
because nodes are often unmatched in capacity. In wired networks, TCP is the most widely used protocol for flow control. TCP is an implicit end to end flow control protocol [6]. The sender maintains a congestion window, limiting the total number of packets that the TCP sender can transmit before receiving acknowledgements. TCP assumes that packet losses are caused by congestion. Therefore when a packet is lost, TCP reduces the size of its congestion window according to the principle of Additive Increase Multiplicative Decrease (AIMD) [7]. Unfortunately, wireless networks suffer from several types of losses that are not related to congestion, making TCP not adapted to this environment. In [8], an enhancement for TCP performance based on Xon/Xoff technique was proposed. Other optimizations and enhancements have been proposed to improve TCP performance in wireless networks $[9,10]$. A synthesis of different TCP improvements in ad hoc networks has been given in [11]. In [12], a state of art on the congestion problem in these networks was given. Studies in $[1,13]$ have shown that an explicit flow control indicating the transmission rate for the sender using feedback is a better alternative for flow control in ad hoc networks.

In this paper, we propose a new intelligent solution to avoid congestion and packets losses. For this, we combine a QoS routing protocol with an explicit flow control mechanism. The proposed QoS routing protocol selects routes having more resources (bandwidth and energy), less delay and better stability. To select the most stable routes, we have proposed a new formula to calculate a link life duration according to the direction, the speed and the energy of nodes. The added flow control mechanism refines the solution by adjusting adaptively the sending rate. Our solution is modeled by ant systems $[14,15]$, which have the potential to address the complex problem. The use of ant systems also reduces congestion by selecting routes in an intelligent manner instead of diffusion.

The remaining part of this paper is organized as follows: Section 2 introduces the related work. In Section 3 we express our motivation. A brief presentation of the ant systems is given in Section 4. Section 5 describes our proposed solution and Section 6 describes our algorithm. Results and their analysis are shown in Section 7. Finally, the last section concludes the paper.

\section{Related Work}

Many studies considered QoS routing and flow control separately in mobile ad hoc networks. Some QoS routing protocols are an extension of existing routing protocols such as: QoS-AODV [16], MP-DSR [17] and QDSDV [18]. Their route discovery method is based on flooding route request packets that cause congestion and affect badly the network performance.

Most QoS routing protocols use only one QoS metric such as: delay [19], link expiration time [20], bandwidth [16, 18] or energy [21]. Others use a combination of multiple QoS routing constraints $[22,23,24,25,26]$. In [22], authors considered the number of hops and the remaining energy in nodes. In $[23,24,25]$, the authors considered the delay and bandwidth. In [26] four metrics are considered: bandwidth, delay, packet loss rate and cost for packet to pass the route. Although in $[23,24,25,26]$, authors considered the bandwidth parameter to choose the route offering more bandwidth to reduce congestion, they did not inform the sender about the adequate transmission rate. Consequently, when a sender transmits with a rate greater than the capacity of at least one of the links in the found route, this link will constitute a bottleneck which causes network congestion.

Flow control is a good mechanism to avoid the congestion problem. In [1], the authors inform the source about the adequate transmission rate, but they considered the routing and the flow control as two independent components. They used DSR protocol [3] as a routing protocol which is based on the choice of the shortest paths. This does not correspond to the best route, in terms of bandwidth, transmission delay, link expiration time or energy. It penalizes applications wishing to transmit with high rate without causing congestion, without frequent disconnections, with minimal delay and with minimal energy consumption.

\section{Motivation}

The performance of the mobile ad hoc networks is strongly influenced by the congestion problem. A congestion control scheme consists of a 
routing algorithm and a flow control scheme. In earlier research, routing and flow control problems have been considered separately. To achieve better performance and better congestion control, routing and flow control must be considered jointly.

In ad hoc networks, most work have been done, essentially, on routing. However many critical parameters such as the bandwidth and the link lifetime are not considered together or are taken with insufficient details. For example, in [20] the authors compute link lifetime without considering nodes energy.

Considering several parameters in routing is an NP-Complete problem. It has been proven that the search of an optimal route satisfying at least two constraints is an NP-Complete problem $[23,28]$. To reduce this complexity, we use ant systems endowed with an intelligence called "swarm intelligence" [15]. These intelligence phenomena in swarms are widely used to solve complex problems [26, 29, 30, 31, 32]. In addition, we have used ant systems for their property, which is the interaction of local nodes, to reduce the amount of control traffic thus reducing congestion, and for their suitability for MANETs characterized by unstable and unpredictable topology. Ant systems offer the means to conceive solutions capable of adapting quickly to changing conditions.

In this study, we propose a novel adaptive and intelligent QoS routing protocol where we give a new combination of critical metrics in ad hoc networks by using ant systems. These metrics are: bandwidth, delay, link expiration time and energy. The choice of the route offering more bandwidth contributes in reducing congestion and packet losses. The choice of delay is important for real time applications and for rapid data transmission. The link expiration time is important for choosing more stable routes. Energy has a huge influence on the network lifetime.

To avoid congestion, we add an explicit flow control mechanism to our QoS routing protocol. This mechanism informs the source about the adequate transmission rate on the found route. This rate corresponds to the smallest bandwidth value for all links constituting the route from the source to the destination.
Before detailing our solution, in the following section we give some basic elements about ant systems.

\section{Ant Systems}

Ant systems appeared towards the end of the nineties in Dorigo's and Colorni's work presented in [14]. In this founder article, they gave a new approach for combinative stochastic optimization and put forward their method's speed to find acceptable solutions while avoiding premature convergence. Optimization by ants' colony is inspired by the ants' behavior during their search for food. They show the way using a volatile chemical substance (which evaporates with time) known as pheromone. The idea in these algorithms is to represent the problem to solve as a search for the best path in a graph. An isolated ant generally moves at random. While moving, it deposits a quantity of pheromones on its trail. Other ants coming behind will choose the most attractive way (with the highest pheromone intensity). When an ant follows a way, it reinforces the trail by adding its own pheromones. The result is a collective behavior at the end of which the more attractive a trail is, the higher is the number of ants that follow it.

To illustrate this behavior, let us consider the experiment shown in Figure 1. A set of ants moves along a straight line from their nest A to a food source B (Figure 1a). At a given moment, an obstacle is put across this way so that side $(\mathrm{C})$ is longer than side (D)(Figure 1b). The ants will thus have to decide which direction they will take: either $\mathrm{C}$ or $\mathrm{D}$. The first ones will choose a random direction and will deposit pheromone

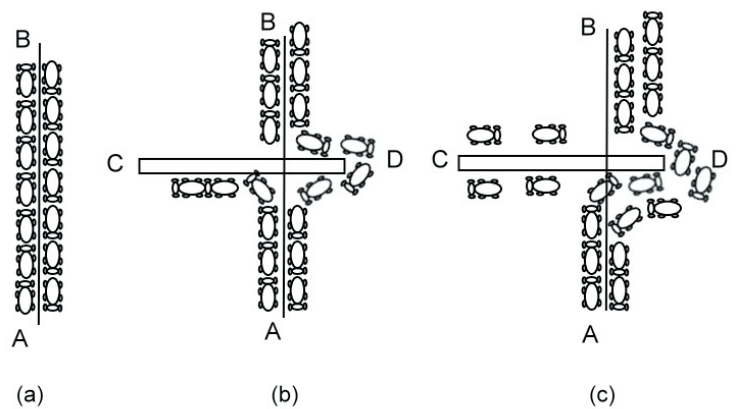

Figure 1. Behaviour of the ants searching the food. 
along their way. Those taking the way ADB (or BDA), will arrive at the end of the obstacle (depositing more pheromone on their way) before those that take the way ACB (or BCA). The following ants' choice is then influenced by the pheromone intensity which stimulates them to choose the path $\mathrm{ADB}$ rather than the way ACB (Figure 1c). The ants will then find the shortest way between their nest and the food source.

This system is modeled by simple and autonomous agents representing artificial ants. In this artificial system, ants are not completely blind. They have a visibility field that affects their displacements. In general, at one point $i$, an ant chooses the point $j$ (i.e. to follow the path $(i, j)$ ) according to the following probability:

$$
P_{i, j}=\frac{\left(\tau_{i, j}\right)^{\alpha} \cdot\left(\eta_{i, j}\right)^{\beta}}{\sum_{(i, k) \in C}\left(\tau_{i, k}\right)^{\alpha} \cdot\left(\eta_{i, k}\right)^{\beta}}
$$

where:

- $\tau_{i, j}$ : is the pheromone intensity on path $(i, j)$.

- $\eta_{i, j}$ : is the ant's visibility field on path $(i, j)$ (an ant assumes that there is food at the end of this path).

- $\alpha$ and $\beta$ : are the parameters which control the relative importance of the pheromone intensity compared to ant's visibility field.

- $\mathrm{C}$ : represents the set of possible paths starting from point $i((i, k)$ is a path of $\mathrm{C})$.

In most cases, an artificial ant will deposit a quantity of pheromone represented by $\Delta \tau_{i, j}$ only after completing their route and not in an incremental way during their advancement. This quantity of pheromone is a function of the found route quality.

Pheromone is a volatile substance. An ant changes the amount of pheromone on the path $(i, j)$ when moving from node $i$ to node $j$ as follows:

$$
\tau_{i, j}=\sigma \cdot \tau_{i, j}+\Delta \tau_{i, j}
$$

Where $\sigma$ is the pheromone evaporation factor. It must be lower than 1 to avoid pheromone accumulation and premature convergence [14].

Several problems were solved by using this method. These problems belong to various domains and their models differ from one problem to another $[29,30,31,32]$.

\section{Solution Description}

We represent the network by a graph $G(V, E)$ where $V$ is the set of mobile nodes in the network and $E$ is the set of its links. We note $\left(v_{i}, v_{j}\right) \in V^{2}$, the link formed by the two nodes $v_{i}$ and $v_{j}$. Our solution takes into account three QoS parameters: the bandwidth $B$, the transfer delay $D$ and the link expiration time $T$. The latter represents the estimated time during which the communication between the two nodes forming the link is always maintained. We thus associate to every link $\left(v_{i}, v_{j}\right)$ a triplet: $B\left(v_{i}, v_{j}\right), D\left(v_{i}, v_{j}\right)$ and $T\left(v_{i}, v_{j}\right)$. Figure 2 illustrates an example of this representation.

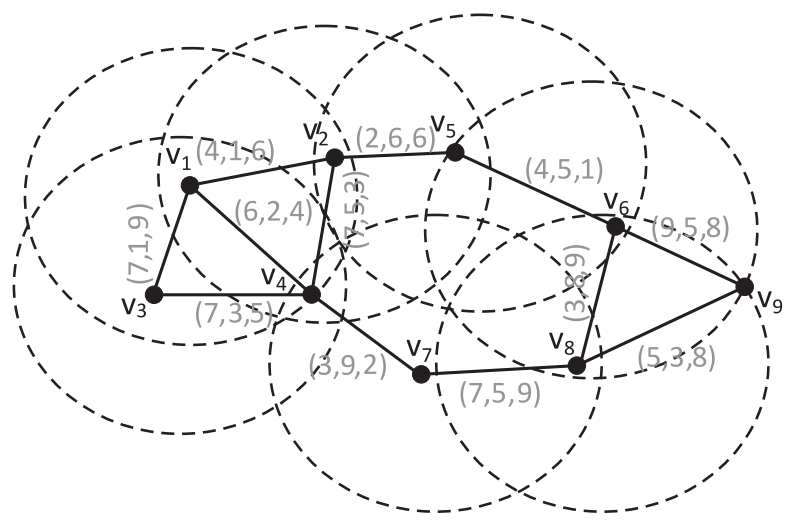

Figure 2. Example of MANET Representation.

A route $R$ is a sequence of nodes starting from a source node and ending at a destination node without any cycle. Routes are built by ants. An ant represents a control packet. To search a route, ants go from the source and pass through intermediate nodes until they reach their destination.

Our solution is composed of two main parts: The first finds the best route insuring a good quality of service; the one that offers higher transmission rate, less delay and more stability (longer link expiration time). The choice of a route offering the highest transmission rate allows the best satisfaction of the user demand in terms of bandwidth, thus avoiding congestion. The choice of a route having a minimum delay allows faster transmission. As for stability, it allows to choose the routes having a longer link expiration time thus avoiding communication interruptions, packets losses and packets 
retransmissions as additional flow. In this part, we have built our QoS routing protocol by using ant systems called "protocol without flow control". In the second part, we have added the flow control mechanism to this latter protocol. This "protocol with flow control" consists of advising the sender about the transmission rate to adapt it to the links capacities within the found route.

To find a route, our routing algorithm uses the pheromone accumulation ants laid on crossed links. This deposited quantity is the same on any link $\left(v_{i}, v_{j}\right)$ along the route $R$. It is noted $\Delta \tau\left(v_{i}, v_{j}\right)$ and it is a function of the global quality of the route $R$. It is expressed by the following equation:

$$
\Delta \tau\left(v_{i}, v_{j}\right)=\frac{B(R)^{\beta_{B}}+T(R)^{\beta_{T}}}{D(R)^{\beta_{D}}}
$$

where:

- $B(R)=\min \left(B\left(v_{i}, v_{i}+1\right), B\left(v_{i}+1, v_{i}+\right.\right.$ 2), .., $\left.B\left(v_{k}-1, v_{k}\right)\right)$; The smallest link bandwidth in route $R$ while $v_{i}$ is the source node and $v_{k}$ the destination node.

- $T(R)=\min \left(T\left(v_{i}, v_{i}+1\right), T\left(v_{i}+1, v_{i}+\right.\right.$ $\left.2), \ldots, T\left(v_{k}-1, v_{k}\right)\right)$; The smallest link expiration time in route $R$.

- $D(R)=D\left(v_{i}, v_{i}+1\right)+D\left(v_{i}+1, v_{i}+2\right)+$ $\ldots+D\left(v_{k}-1, v_{k}\right)$; The sum of delays on all links in route $R$.

- $\beta_{B}, \beta_{T}$ and $\beta_{D}$ denote the link weight factors that show the relative significance of each QoS parameter during pheromone update on a route $R$. Although the pheromone is deposited on the link, its quantity (equation 3 ) is only defined after finding the route. It permits to appreciate in the same way all links forming the route. Nevertheless, the local quality of a link is taken into account in its choice. This local quality represents the heuristic factor or the visibility of the ant. It is given by the following equation:

$$
\eta_{i, j}=\frac{B\left(v_{i}, v_{j}\right)_{B}^{\alpha}+T\left(v_{i}, v_{j}\right)_{T}^{\alpha}}{D\left(v_{i}, v_{j}\right)_{D}^{\alpha}}
$$

Where $\alpha_{B}, \alpha_{T}$ and $\alpha_{D}$ are parameters showing the relative importance of each QoS parameter during the link selection.

When an ant searches for a route, it chooses probabilistically one node among its neighbour's nodes that are not visited yet. The routing probability value between $v_{i}$ and $v_{j}$ is computed by the composition of the strength of pheromone values (equation 6) and the visibility values (equation 4).

$$
P_{i, j}=\frac{\left[\tau\left(v_{i}, v_{j}\right)\right]^{\alpha} \cdot\left[\eta_{i, j}\right]^{\beta}}{\sum_{k \in M}\left[\tau\left(v_{i}, v_{k}\right)\right]^{\alpha} \cdot\left[\eta_{i, k}\right]^{\beta}}
$$

where:

- $\tau\left(v_{i}, v_{j}\right)$ is the amount of pheromone on the link $\left(v_{i}, v_{j}\right)$

- $\eta_{i, j}$ is the visibility of the link $\left(v_{i}, v_{j}\right)$

- $\alpha$ and $\beta$ are two parameters that show the relative significance of the pheromone and the visibility during the process of QoS route discovery.

- $\mathrm{M}$ : is the set of all possible neighbor nodes $v_{k}$, not visited yet by the ant.

The pheromone quantity on the link $\left(v_{i}, v_{j}\right)$ is:

$$
\tau\left(v_{i}, v_{j}\right)=\rho \cdot \tau\left(v_{i}, v_{j}\right)+\Delta \tau\left(v_{i}, v_{j}\right)
$$

where $\rho$ is the evaporation factor $(0<\rho<1)$. It is used to avoid the infinite increment of pheromone on the link that may lead to stagnation route.

When an ant reaches the destination, a route is found. Then, the destination node sends a backward ant to indicate this route for the sender. It also informs it about the transmission rate in the case where the flow control mechanism is integrated.

\subsection{Used QoS Parameters}

- The Bandwidth: Represents the transmission link capacity. The link having the largest bandwidth is always chosen in our process of the route discovery. Several approaches have been used to estimate the bandwidth $[25,33,34]$. We have used the approach presented in [25] because it does not require any supplementary traffic.

- Delay: The delay of a link is given by the difference between the instant a HELLO packet is created and the instant of its receipt by the neighbouring node [25]. 
- Link Expiration Time: The link expiration time $T\left(v_{i}, v_{j}\right)$ is an important parameter to consider in mobile networks. In $[35,36]$, the authors considered the extreme case where the two nodes move away one from the other. However, there can be some situations where the two mobile nodes come closer to one another or go in the same direction with the same speed. It makes the link more attractive because it is maintained for a long time. In [20], the authors define the time during which two mobile nodes remain connected (at a distance lower or equal to the transmission range) according to their directions and their speeds. The equation below gives the expiration time (stability) of a link formed by nodes $v_{i}$ and $v_{j}$ having the coordinates $\left(x_{i}, y_{i}\right)$ and $\left(x_{j}, y_{j}\right)$, the speeds $S_{i}$ and $S_{j}$ and the directions $\theta_{i}$ and $\theta_{j}\left(0<\theta_{i}, \theta_{j}<2 \Pi\right)$ respectively.

$$
\begin{aligned}
& T^{\prime}\left(v_{i}, v_{j}\right)= \\
& =\frac{-(a b+c d)+\sqrt{\left(a^{2}+c^{2}\right) r^{2}-(a d-b c)^{2}}}{a^{2}+c^{2}}
\end{aligned}
$$

where:

- $a=S_{i} \cos \theta_{i}-S_{j} \cos \theta_{j}$

- $b=x_{i}-x_{j}$

- $c=S_{i} \sin \theta_{i}-S_{j} \sin \theta_{j}$

- $d=y_{i}-y_{j}$

- $r=$ Transmission range of each node.

The parameters considered in this formula are not sufficient to correctly determine the link expiration time. In fact, this latter does not depend only on nodes mobility; it is not sufficient to have two nodes in the same transmission range to say that the link between them is maintained. The link expiration time depends also on energy sources life duration of nodes. According to equation (7), when $S_{i}=S_{j}$ and $\theta_{i}=\theta_{j}$ the life duration of the link $\left(v_{i}, v_{j}\right)$ becomes infinite $(\infty)$. This cannot be true when considering the energy constraint, which has a great influence on the links life (duration) in MANETs.

In this work, we include energy in computing the link expiration time. For a link $\left(v_{i}, v_{j}\right)$, the link expiration time is the minimum between its life duration according to equation(7) and the minimal duration in autonomy of the two nodes $v_{i}$ and $v_{j}$. It is given by the following equation:

$$
T\left(v_{i}, v_{j}\right)=\operatorname{Min}\left[T^{\prime}\left(v_{i}, v_{j}\right), \operatorname{Min}\left(E\left(v_{i}\right), E\left(v_{j}\right)\right)\right]
$$

Where $E\left(v_{i}\right)$ and $E\left(v_{j}\right)$ are the life duration of nodes energy.

\section{Algorithm Description}

The proposed algorithm is distributed on all nodes of the network. Each node $v_{i}$ maintains tables: A table of Pheromones "Pherotab" specifying the quantity of available pheromone on each link $\left(v_{i}, v_{j}\right)$, (equation 6$)$, this quantity is initialized to a constant $\mathrm{C}$, and the table of Probabilities "Probtab" indicating its transition probabilities towards its neighboring nodes $v_{j}$ (equation 5). All tables of the nodes forming the route are updated when the ant returns from the destination node to the source node.

An ant is a small packet having, among others, the following fields:

- Visited: Is the list of the nodes that are progressively visited by the ant. This list forms the route $R$ from the source node to the destination node.

- Rate: Contains the minimum transmission rate on the found route. Once the ant has reached the destination, this field will contain the smallest transmission rate of the route. It will be returned to the source to regulate its transmission rate, in the case where the mechanism of flow control is added.

- Delay: Keeps the sum of delays on all route links (delay of the route).

- Stab: Contains the minimal link expiration time on the route links (life duration or stability of the route).

- Visib: Contains the list of the visibility factors of the ant on every crossed link. Used to update transition probabilities.

- Sumphero: Represents the quantity of accumulated pheromones on the route. This quantity divided by the number of hops in the route determines its quality. It is initialized to zero.

- TTL: Time To Live of the ant. The time after which the ant will be killed. 
To determine each node neighbors a HELLO packet is used. It allows the nodes receiving it to declare the sender as a neighbor node. It also has a field containing the instant of its creation allowing the link delay calculation.

Our algorithm is composed of two major procedures:

The first procedure gives the route discovery process by the ants. When a source node initiates a routing request, it sends $N$ ants periodically. As described below:

Procedure Forward-Ant (SourceNode,

DestinationNode, Visited, Visib, Rate, Stab,

Delay) \{

1. Visited $\leftarrow$ SourceNode //list of visited nodes initialized to source node

2. Pherotab $\leftarrow \mathrm{C} / /$ all network links are initialized to $\mathrm{C}$ value of pheromone

3. Initially choose a node $\mathrm{K}$ randomly

4. While $(\mathrm{K} \neq$ DestinationNode $)\{$

5. If $(\mathrm{K} \in$ Visited $)\{/ /$ has this node already visited

6. Delete from visited all nodes starting from the node $\mathrm{K}$ until the end of visited.

//This is crucial to avoid routing loops.

7. Choose another node.

\} Else \{

8. Visited $\leftarrow \mathrm{K} / /$ add this node to the list of visited nodes

9. Compute the visibility for this link using equation(4)

10. Insert the visibility result to Visib list.

11. Rate $\leftarrow \min ($ Rate, Rate of this link)

12. $\operatorname{Stab} \leftarrow \min ($ Stab,Stability of this link)

13. Delay $\leftarrow$ Delay + Delay of this link

14. Choose a new node $\mathrm{L}$

15. $\mathrm{K} \leftarrow \mathrm{L}$

\}//else

\}//end of while; the destination node is reached.

16. Compute the quantity of pheromone

$\Delta \tau\left(v_{i}, v_{j}\right)$ to deposit on the links of the route.

\}$/ /$ end of the procedure.

When a destination node has received an ant $M(M<N)$ the second procedure is executed.
This procedure sends back the ant to its source informing it about the found route. It is described below:

Procedure Backward-Ant (Visited, Visib,

Sumphero $\{$ // Sumphero initialized to zero

1. Compute the reverse path from the forward path stored in Visited

2. $\mathrm{F} \leftarrow$ DestinationNode

3. Send this ant to the source node

4. While $(\mathrm{F} \neq$ SourceNode $)\{$

5. Update Probtab according to equation

(5) $/ /$ probabilities tables

6. Update Pherotab according to equation

(6) $/ /$ the pheromones tables

7. Sumphero $\leftarrow$ Sumphero + pheromone on this link

8. $\mathrm{F} \leftarrow$ the next node in the reverse path

\} // end of while

\}$/ /$ end of the procedure.

This code illustrates our routing algorithm without flow control mechanism. To consider the explicit flow control mechanism, to the returning ant packet we add the field Rate that contains the rate value with which the source node must transmit its data. For this, a supplementary code is also added to force the sender to transmit its packets with the rate indicated in the Rate field.

\section{Simulations and Results}

\subsection{Simulation Configurations}

Our work is carried out by using Network Simulator (NS2.31) [37]. We use the IEEE 802.11 Distributed Coordination Function (DCF) in MAC layer. The used mobility model is the Random Waypoint Model [38], where each node independently chooses a random initial point and waits there for a period called pause time. Then it moves with a velocity chosen uniformly between minimum and maximum velocities to a randomly chosen destination. After reaching the destination, it waits again for the pause time and then moves to a new randomly chosen destination with a new chosen velocity. Each node repeats independently this movement 
through the simulation. The simulation time is 300 seconds. The traffic enters the simulated network with packets size of 512 bytes. We performed a set of simulations to evaluate the performance of our protocol with and without flow control compared to QoS-AODV protocol. The two protocols, QoS-AODV and our QoS protocol without flow control send the traffic at a rate of 5 packets per second. Each scenario uses the same protocol parameters. The values of $\alpha, \beta, \beta_{B}, \beta_{T}, \beta_{D}, \alpha_{B}, \alpha_{T}, \alpha_{D}$, the initial pheromone value $C$ and the value of the pheromone factor decay $\rho$ are given in Table 1. Though the setting for these parameters is very important, it is still difficult to find a suitable method. We have used the traditional way to set these parameters; the values are chosen, after testing several values for each of them. Marco Dorigo and al. [14] set experimentally the ant algorithm parameters for the travelling salesman problem. However, Marcin L. Pilat and Tony White [39] use genetic algorithms to set the ant algorithm parameters for the same problem.

\begin{tabular}{|c|c|c|c|c|c|c|c|c|c|}
\hline$\alpha$ & $\beta$ & $\beta_{B}$ & $\beta_{T}$ & $\beta_{D}$ & $\alpha_{B}$ & $\alpha_{T}$ & $\alpha_{D}$ & $\mathrm{C}$ & $\rho$ \\
\hline 0.5 & 0.5 & 0.9 & 0.7 & 0.4 & 0.9 & 0.7 & 0.4 & 0.5 & 0.3 \\
\hline
\end{tabular}

Table 1. Simulation parameter setting.

\subsection{Performance Metrics}

To evaluate the performance of our protocol with and without flow control, we use three performance metrics:

- Packet Delivery Ratio: Is the fraction of total number of packets received by the destination nodes to the total number of packets sent by the source nodes.

- Average End to End Delay (Average ETE Delay): is the total time needed to deliver successfully a packet by a source node till it is received by the corresponding destination node.

- Average Energy Consumption: is the total network energy consumed over the number of nodes.

\subsection{Results and Discussions}

The presented results are the average of 100 individual runs. We first discuss the performance of the algorithm in terms of packet delivery ratio versus mobility. Two sets of simulations are performed with 60 nodes in an area of $1000 \mathrm{~m} \mathrm{X}$ $1000 \mathrm{~m}$. The first one is performed with a minimum velocity of $1 \mathrm{~m} / \mathrm{s}$ and a maximum velocity of $2 \mathrm{~m} / \mathrm{s}$ and the second one is performed with a minimum velocity of $3 \mathrm{~m} / \mathrm{s}$ and a maximum velocity of $10 \mathrm{~m} / \mathrm{s}$. For both sets, simulations are performed with 8 different pause times: 0 , $25,50,100,150,200,250$ and 300 seconds to simulate different mobility patterns. Results are shown in Figure 3 and Figure 4.

In Figure 3, we notice that the more the pause time increases, which presents smaller nodal motion, the more the data delivery ratio increases for the three protocols. For QoS-AODV the data delivery ratio is about $82 \%$. Our protocol (with or without flow control) presents better results. The packet delivery ratio for our protocol without flow control is about $85 \%$ and about $90 \%$ when using flow control. In the case of the protocol without flow control, the improvement is due to the fact that the chosen routes are those having higher bandwidth and a longer link expiration time. This reduces

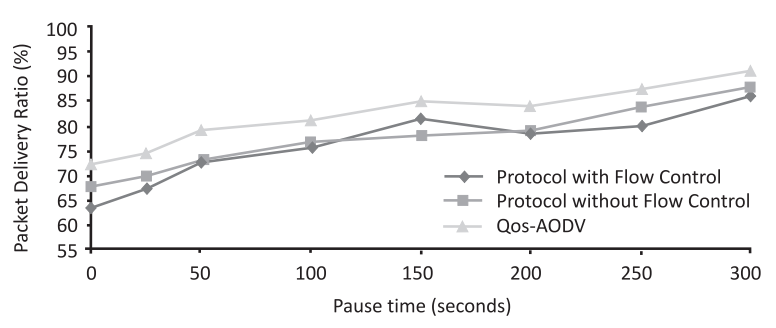

Figure 3. Simulation of 60 nodes in the area of $1000 \mathrm{~m} \times 1000 \mathrm{~m}$ with $1 \mathrm{~m} / \mathrm{s}<=$ speed $<=2 \mathrm{~m} / \mathrm{s}$.

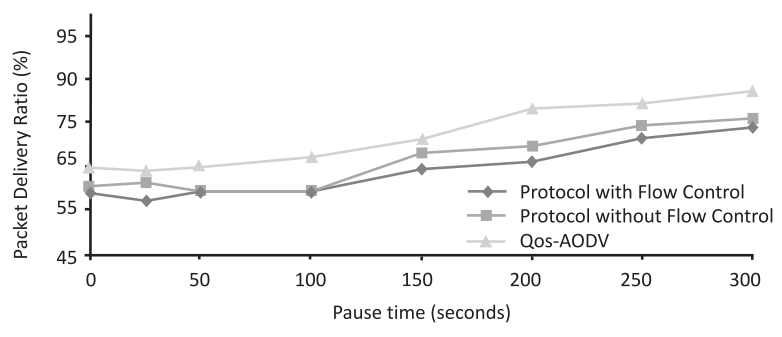

Figure 4. Simulation of 60 nodes in the area of $1000 \mathrm{~m} \times 1000 \mathrm{~m}$ with $3 \mathrm{~m} / \mathrm{s}<=$ speed $<=10 \mathrm{~m} / \mathrm{s}$. 
congestion and frequent disconnections, therefore losses are reduced consequently. When a flow control mechanism is added, the result is improved again due to the fact that the source always transmits with a rate that doesn't exceed the capacity of all links forming the route.

Figure 4 shows results for the second set of simulations. The curves of this figure have the same trend as those of Figure 3; as the pause time gets more intensive, the packet delivery ratio increases. Compared to the results in Figure 3 , we notice that the performance gets worse for the three protocols. This is mainly due to the nodes speed increase.

Secondly, we have studied the impact of pause time variation on average end-to-end delay. We used 60 nodes in a square area $(1000 \mathrm{~m} \times 1000 \mathrm{~m})$ with $10 \mathrm{~m} / \mathrm{s}$ as a maximal speed.

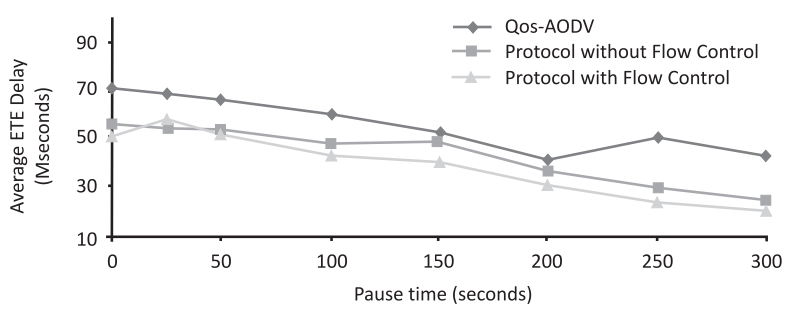

Figure 5. Average ETE delay (ms) vs pause time (s).

Results in Figure 5 show that the average endto-end delay increases with the nodal motion increase. When the pause time is null, i.e. the nodes are in continual movement, the delay reaches a higher value for the three protocols. But when the nodes use a higher pause time, this delay decreases. When the nodes are immobile (pause time $=300 \mathrm{sec}$ ) the smallest values are obtained by all the protocols. This figure shows that the average end-to-end delay of QoSAODV is more important than that presented by our protocol. This is due to the fact that QoS-AODV relies on just one route. Therefore, when this route becomes invalid, packets wait until a new route is found. Our protocol (with or without flow control) improves this end-to-end delay for various reasons: - the chosen routes are always those presenting a minimum delay, - and those having longer link expiration time (equation 6). We also note that the average endto-end delay of the protocol with flow control is better. This is because, the transmission rate of the source nodes does not exceed the capacities of the links forming the routes; this avoids packets waiting in queues.

Finally, in Figure 6 we explore the impact of mobility on average consumed energy. Simulation environment is similar to that of previous simulations.

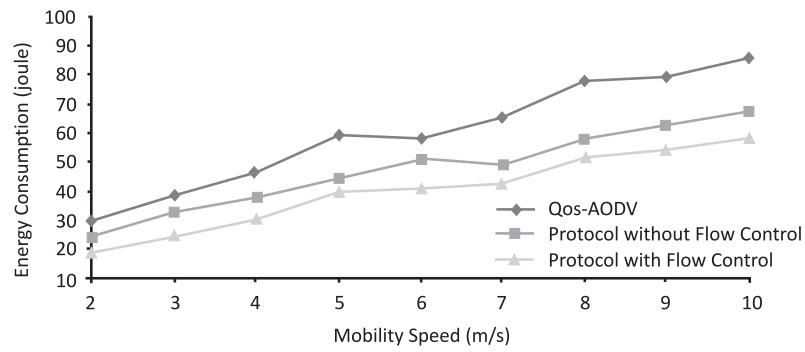

Figure 6. Average energy consumption (joule) vs mobility speed $(\mathrm{m} / \mathrm{s})$.

Figure 6 shows that the average consumed energy increases with the increase of speed for the three protocols. However, the QoS-AODV is the protocol that consumes the most, especially for speeds higher than $5 \mathrm{~m} / \mathrm{s}$. This is due to packets retransmissions by QoS-AODV after their loss and to the fact that QoS-AODV broadcasts control packets while searching for a route that consumes more energy. However, our protocol without or with flow control, uses another mechanism to discover a route. It sends ants and each one chooses a next node in a probabilistic manner. This reduces the number of control packets that are sent, and consequently less energy will be consumed. As the protocol with the flow control adapts its transmission rate to the route capacity, the number of lost packets is reduced. Therefore, less packets retransmission are carried out, which explains that the protocol with flow control is the one consuming less energy.

\section{Conclusion}

Various routing and flow control protocols are often considered and implemented separately. In this paper, we have combined a flow control mechanism with the QoS routing protocol. The proposed solution improves the network performance by finding the better route with larger bandwidth, smaller delay and better stability. In order to assess the link stability, we have 
proposed a new metric that takes into account direction, speed and energy of the nodes. The added flow control mechanism allows rate adjustments for each link which further improves the performance. Using NS2 Network Simulator, the proposed protocol (with and without the flow control) was compared with other protocols such as QoS-AODV. Our numerical results confirm that the proposed protocol outperforms the other protocols. In our future work, we will study a non-cooperative environment where some nodes are selfishly refusing to forward packets to other nodes.

\section{References}

[1] KAI CHEN \& AL., The Utility of Explicit Rate-based Flow Control in Mobile Ad Hoc Networks. WCNC 2004, March 2004.

[2] C. E. Perkins, E. M. Belding-Royer And S. DAS, Ad Hoc On-demand Distance Vector (AODV) Routing. IETF RFC 356.

[3] D. Johnson \& AL., Dynamic Source Routing in Ad Hoc Wireless Networks. In IETF Internet Draft, March 2003.

[4] T. Clausen AND P. JACQuet, Optimized Link State Routing Protocol. In IETF Internet Draft, July 2003.

[5] S. Chakrabarti, A. Mishra, QoS issues in ad hoc wireless networks. Communications Magazine, IEEE, Vol. 39, No. 2, pp. 142-148, 2001.

[6] RFC 793, Transmission Control Protocol, September 1981 .

[7] D. CHIU AND R. JAIN, Analysis of the Increase/Decrease Algorithms for Congestion Avoidance in Computer Networks. Journal of Computer Networks and ISDN, Vol. 17, No. 1, June 1989, pp. $1-14$.

[8] M. Belkadi, M. Lalam, A. M'zoughi, R. AoudJIT, M. DAOUI, K. AIT ALI, Amélioration des Performances de TCP dans les Manets par la Technique Xon/Xoff. 9ème Conférence Magrébine sur les Technologies de l'information, MCSEAI'06 Proceedings, pp. 86-90.

[9] F. WANG AND Y. ZHANG, Improving TCP Performance over Mobile Ad Hoc Networks with Out of Order Detection and Response. MOBIHOC'02, June 9-11, 2002, EPFL Lausane, Switzerland.

[10] K. DONGKYUN \& AL., TCP-BuS: Improving TCP Performance in Wireless Ad Hoc Networks. Journal of Communications and Networks, Vol. 3, No. 2, June 2001.

[11] A. Al Hanbali \& AL., A Survey of TCP over Mobile Ad Hoc Networks. IEEE Communications Surveys and Tutorials, Vol. 7, No. 3, pp. 22-36, August 2005.
[12] Christian LOCHERT, BJOM SCHEUERMANN, MARTIN MAUVE, A Survey on Congestion Control for Mobile Ad Hoc Networks. Wiley Wireless Communications and Mobile Computing 7(5), pp. 655-676, June 2007.

[13] K. Sundaresan \& AL., ATP: A Reliable Transport Protocol for Ad Hoc Networks. Mobihoc'03, June 2003.

[14] M. DoRigo \& AL., The Ant System: Optimization by a colony of cooperating agents. IEEE Transactions on Systems, Man and Cybernetics, Part B, Vol. 26, No. 1, pp. 29-41, 1996.

[15] ERIC Bonabeau AND GuY Theraulaz, L'intelligence en essaim. Pour la Science, (271) pp. 66-73, May 2000.

[16] C. E. Perkins, E. M. Belding-Royer, Quality of Service for Ad Hoc Distance Vector Routing. IETF Internet Draft, draft-perkins-manet-aodvqos-00.txt, November 2001.

[17] R. LEUNG \& AL., MP-DSR: A QoS-Aware MultiPath Dynamic Source Routing Protocol for Wireless Ad-Hoc Networks. 26th Annual IEEE Conference on Local Computer Networks (LCN), Tampa, Florida, USA, November 2001.

[18] T.-W. Chen, J. T. Tsai And M. Gerla, QoS Routing Performance in Multihop, Multimedia, Wireless Networks. IEEE International Conference on Universal Personal Communications'97, pp. 451-557, October 1997.

[19] A. Munaretto, H. Badis, K. Al Agha And G. PUJOLLE, QOLSR: Routage avec QoS dans OLSR. AlgoTel, Banyals-sur-mer, France, May 2003, INRIA.

[20] WILliaM SU \& AL., Mobility prediction in wireless networks. Proceedings of IEEE MILCOM 2000, Los Angeles, CA, October 2000, pp. 491-495.

[21] JIN-MAN KIM AND JONG-WoOK JANG, AODV based Energy Efficient Routing Protocol for Maximum Lifetime in MANET. Proceedings of the Advanced International Conference on Telecommunication and International Conference on Internet and Web Application and Service (AICT/ICIW 2006).

[22] O. Hussein AND T. SAADAWI, Ant Routing for Mobile Ad Hoc networks (ARAMA). 2004, IEEE, pp. 281-285.

[23] Zheng WANG AND Jon CROWCROFT, Quality-ofService Routing for Supporting Multimedia Application. IEEE Journal on Selected Areas in Communications, Vol. 14, No. 7, September 1996.

[24] Peng. Fu and Deyun Zhang, Hybrid Optimize Strategy-based QoS Route Algorithm for Mobile Ad Hoc Networks. Journal of Computer Science 2(2): pp. 160-165, 2006.

[25] H. BADIS AND K. Al AgHA, QoS for ad hoc networking based on multiple-metrics: Bandwidth and delay. IFIP MWCN'03: Mobile and Wireless Communications Networks, Singapore, October 2003. 
[26] Peng Fu and Deyun Zhang, A Heuristic and Distributed Qos Route Discovery Method for Mobile Ad Hoc Networks. WAIM 2005, LNCS 3739, pp. 428-439, October 2005.

[27] A. COLORNI, M. DORIGO, V. MANIEZZO, Distributed Optimization by Ant Colonies. Proceedings of ECAL91 - European Conference on Artificial Life, Paris, France, Elsevier Publishing, pp. 134-142, 1991.

[28] J. M. JAFFE, Algorithms for Finding Paths with Multiple Constraints. Networks, Vol. 14, No. 1, pp. 95-116, 1984.

[29] M. Dorigo And L. M. GAmbardella, Ant colony system: a cooperative learning approach to the travelling salesman problem. IEEE Transaction on Evolutionary Computation, 1(1), 1997.

[30] I. Alaya, C. Solnon, K. Ghedira, Optimisation par colonies de fourmis pour le problème du sac ŕ dos multi-dimentionnel. Techniques et Sciences Informatiques (TSI) 26(3-4): pp. 371-390, HermèsLavoisier 2007.

[31] YAN TIAN \& AL., Dynamic Vehicle Routing Problem Using Hybrid Ant System. In IEEE Intelligent Transportation Systems, Vol. 2, pp. 970-974, 2003.

[32] ZHENYU LIU, MARTA Z. KWIATKOWSKA, AND COSTAS CONSTANTINOU, A Biologically Inspired Qos Routing Algorithm for Mobile Ad Hoc Networks. International Journal of Wireless and Mobile Computing (IJWMC) 2006.

[33] Samarth H. Shah, Kai Chen, Klara NahrstEDT, Available Bandwidth Estimation in IEEE 802.11 Based Wireless Networks. Proceedings of the first ISMA/CAIDA Bandwidth Estimation Workshop (Best 2003), SansDiego, California, USA, December 2003.

[34] KaZANTZIDIS AND M. Gerla, End-to-end versus Explicit Feedback Measurement in 802.11 Networks. In Seventh IEEE Symposium on Computers and Communications, 2002.

[35] K. WU AND J. HARMS, Location trace aided routing in mobile ad hoc networks. In Proceedings of the 9th International Conference on Computer Communications and Networks (IC3N), pp. 354-359, October 2000.

[36] S. K. DAS, A. MuKHERJEE, S. BANDYOPADHYAY, K. PAUL, D. SAHA, Improving quality-of-service in ad hoc wireless networks with adaptive multi-path routing. Proceeding of IEEE GLOBECOM, San Francisco, November 2000, pp. 261-265.

[37] The Network Simulator (NS2). http://www.isi.edu/nsnam/ns/.

[38] JungKeun Yoon \& AL., Random Waypoint Considered Harmful. IEEE INFOCOM, 2003.

[39] Marcin L. Pilat and Tony White, Using Genetic Algorithms to Optimize ACS-TSP, Lecture Notes in Computer Science, Vol. 2463. Proceedings of the Third International Workshop on Ant Algorithms, pp. 282-287, 2002.
Received: April, 2009 Revised: November, 2009 Accepted: November, 2009

Contact addresses:

M. Belkadi

Computer Science Department Tizi Ouzou University, Algeria e-mail: belkadi2005@yahoo.fr

A. M'zoughi IRIT UPS 118 Route de Narbone 31062 Toulouse Cedex France

MALIKA BELKADI is an Assistant Professor of electrical engineering at the Faculty of Electrical Engineering, University of Tizi Ouzou. She is also a research member at the LARI Laboratory of the Computer Science Department. Her areas of interest include mobile networks, and embedded systems and computer architecture.

MUSTAPHA LALAM received the Master's degree in computer architecture from the High School of Computer Science, Algiers, Algeria, in 1980 and the Ph.D. degree in computer science from the University of Toulouse, France, in 1990. He joined the University of Tizi Ouzou, Algeria in 1993, where he is now Professor in the Computer Science Department at the University of Tizi Ouzou. He has been involved in research and development of computer architecture, distributed systems and mobility management for wireless mobile computing and communications.

ABdelaziz M'zoughi is a Professor at Computer Science Institute, University of Toulouse, France. His areas of interest include mobile networks, computer architecture and embedded systems.

NoURDINE TAMANI received the Master's degree from the University of Tizi Ouzou. Currently, he is a Ph.D. student of computer science at IRISA, Rennes I University. His areas of interest include the ITS: intelligent transportation systems and mobile networks.

MEHAMMED DAOUI is an Assistant Professor of electrical engineering at the Faculty of Electrical Engineering, University of Tizi Ouzou. He is also a research member at the LARI Laboratory of Computer Science Department. His areas of interest include mobile networks, and embedded systems.

RACHIDA AOUDJIT is an Assistant Professor of electrical engineering at the Faculty of Electrical Engineering, University of Tizi Ouzou. She is also a research member at the LARI Laboratory of the Computer Science Department. Her areas of interest include mobile networks, computer architecture and embedded systems. 
